\title{
Gas Sensors for Indoor Air Quality
}

\author{
D. Puglisi ${ }^{1}$, J. Eriksson ${ }^{1}$, M. Bastuck ${ }^{1,2}$, M. Andersson ${ }^{1,3}$, A. Schütze ${ }^{2}$, A. Lloyd Spetz ${ }^{1,3}$ \\ Applied Sensor Science, IFM, Linköping University, 58183 Linköping, Sweden \\ ${ }^{2}$ Lab for Measurement Technology, Saarland University, 66123 Saarbrücken, Germany \\ ${ }^{3}$ Microelectronics and Materials Physics Laboratories, University of Oulu, P.O. Box 4500, 90014 Oulu, \\ Finland \\ Corresponding author: donatella.puglisi@liu.se
}

\begin{abstract}
This study is a short overview on air pollution, its definition, history, and adverse effects on health, environment, and economy, with a particular emphasis on indoor air quality. We demonstrate that gas sensors based on silicon carbide field effect transistor (SiC-FET) technology are suitable for indoor air quality applications due to their high sensitivity, long-term stability, and robustness. Enhancement of selectivity can be reached through the optimization of the sensing layer (gate contact) in combination with smart operation and multivariate statistical methods for data analysis. Formaldehyde, naphthalene, and benzene, belonging to the class of hazardous volatile organic compounds (VOCs), are our target gases at the low parts per billion concentration level.
\end{abstract}

Key words: air pollution, indoors, volatile organic compounds, gas sensors, silicon carbide field effect transistors (SiC-FETs)

\section{A Glance at the History of Air Pollution}

Air pollution is the degradation of air quality due to harmful chemicals or other substances.

The first and greatest air pollution disaster of Earth's history occurred during the Proterozoic era, between 2.5 and 0.5 billion years ago, when the atmosphere changed from devoid of oxygen to oxygen-rich [1]. Emissions of large quantities of oxygen, a highly reactive and therefore toxic air pollutant at that time, were due to the rise of early photosynthetic plant life, and caused many established primitive life forms to go extinct. On the other hand, an oxygen-rich atmosphere allowed the rise of many new forms of life, such as complex plants and animals. Since then ( 0.5 billion years ago), the atmospheric oxygen levels have oscillated around the current level $(20.9 \%$ at sea level under dry conditions) for hundreds of millions of years.

A schematic representation of geologic time from the Earth's formation around 4.5 billion years ago to the present is shown in Fig. 1. As shown in Fig. 1, 4 to 6 million years ago, human ancestors learned to use and, eventually, control fire. Early burning of organic fuels caused the first indoor air quality issues of human history. Our ancients in caves or other early dwellings for sure experienced the acute effects of irritating smokes, but most probably they could not link indoor air quality to chronic health effects. The first awareness of adverse health effects due to air pollution arrived during the $13^{\text {th }}$ century with the introduction of coal as a new fuel. The widespread availability of this fuel for both industrial and domestic uses led to increasingly polluted indoor and outdoor air. Consequently, the first regulatory actions arose. The impact of the Industrial Revolution brought about regulations in Great Britain (the first Public Health Act was introduced in 1848) and USA (local ordinances were issued from the 1880s) aiming at controlling air pollutant emissions, smoke and ash in particular. However, little was done to effectively control air pollution due to the pressure for progress and its many associated benefits. Only the great pollution disasters of the $20^{\text {th }}$ century changed the way to perceive and address to the society the adverse effects of air pollution.

Nowadays, there are several national and international policies for reducing exposures and controlling emissions, and for setting limits and target values for air quality, but further joint efforts are still needed. Technological solutions, laws and regulations, raising awareness and changing people's behavior are all important measures.

We need a sustainable environment for the future generations and ourselves, which has to be healthy, comfortable, and energy-efficient. 
It is therefore necessary to develop effective solutions to efficiently monitor and control air quality, both indoors and outdoors, while containing costs.

Our contribution to reach this goal consists of the development of reliable and cost efficient gas sensors based on silicon carbide field effect transistor (SiC-FET) technology for highly sensitive and selective detection of three hazardous volatile organic compounds (VOCs), i.e., formaldehyde, naphthalene, and benzene, at low parts per billion ( $p p b)$ levels.

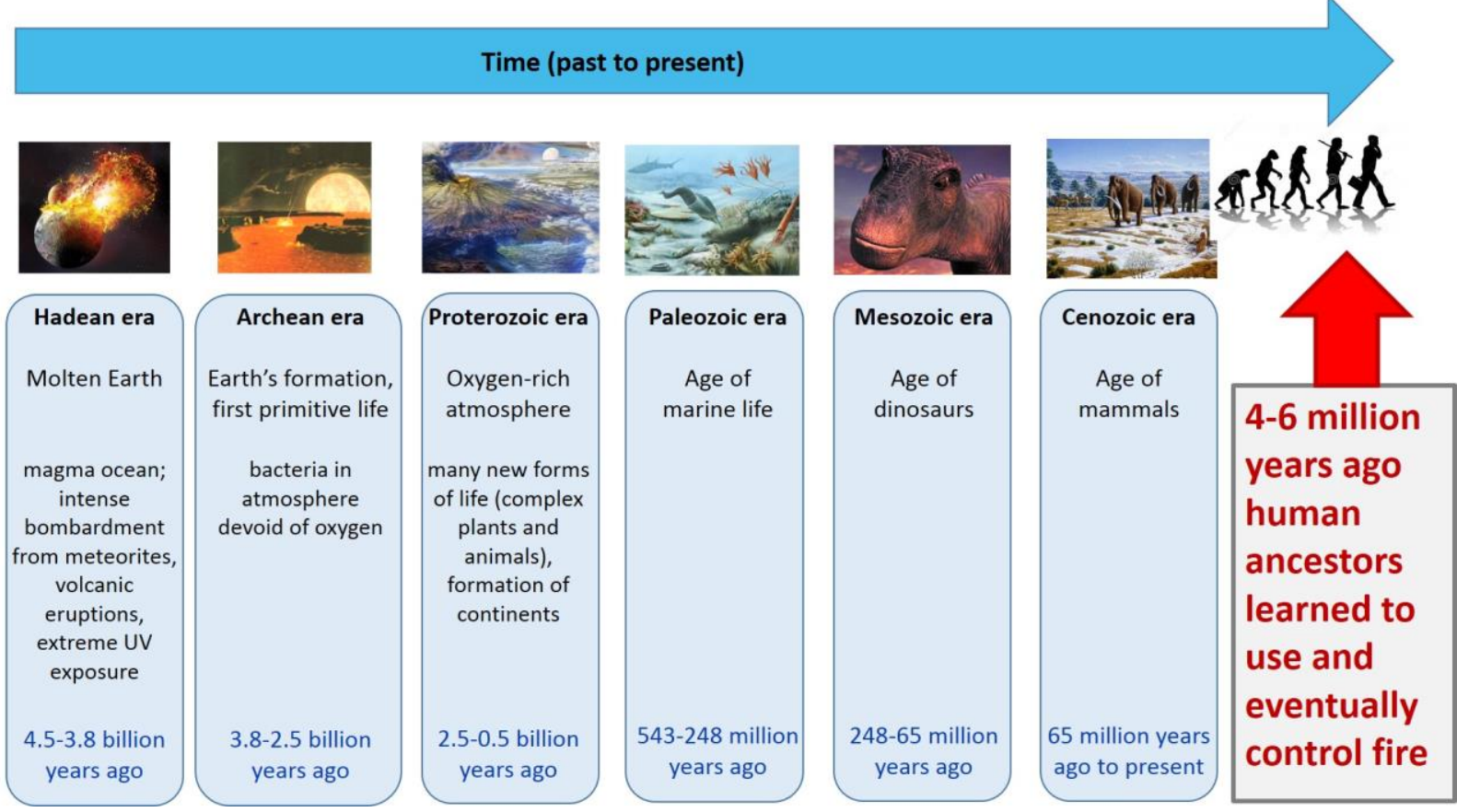

Fig. 1. Geologic Time from the Earth's formation (Hadean era) to present (Cenozoic era). The figure is adapted from [1].

\section{Volatile Organic Compounds and Indoor Air Pollution}

Volatile organic compounds (VOCs) belong to a wide class of chemicals containing carbon, hydrogen (pure hydrocarbons) and, possibly, oxygen (e.g. aldehydes) or other elements. Although the term VOC is used for both indoor and outdoor air quality, the definition and the emphasis given to the concern outdoors is different from indoors. The U.S. Environmental Protection Agency (EPA) regulates VOCs outdoors mainly because of their ability to create photochemical smog under certain conditions. The main concern for indoor environment, instead, is the potential adverse impact on the health of people that are exposed. For indoor air quality, all organic chemical compounds whose compositions give them the potential to evaporate under normal atmospheric conditions are considered as VOCs [2]. These are major air pollutants in various indoor environments, and around 200 among them have been classified as possible, probable, or known human carcinogens.

According to the EPA, indoor air is, on average, two to five times more polluted than outdoor air, and in the worst cases even 100 times. This is because confined areas enable potential pollutants to build up more easily than open spaces do. In cold climates or during winters, the problem appears to be more serious because of the higher number of hours spent indoors and lower air exchange rate due to cold temperature and energy conservation concerns. Furthermore, in highly urbanized and industrial areas, inadequate ventilation and high levels of humidity can increase concentrations of indoor air pollutants.

\section{Giving Numbers}

Exposure to air pollution has emerged as the fourth important risk factor for deaths worldwide right after metabolic risks, dietary risks, and tobacco smoke [3]. Almost $3 \%$ of the total burden of disease is due to indoor air pollution, as reported by the World Health Organization (WHO). Illnesses related to poor indoor air quality include a number of symptoms ranging from headache, nausea, dizziness, and faster skin aging to asthma or other acute or chronic respiratory problems, allergies or even cancer. Indeed, it has been estimated that $80 \%$ of all cancers are attributed to environmental rather than to genetic factors, including exposure to carcinogenic chemicals, many of which are found in household cleaning products, as confirmed by the International Agency for 
Research on Cancer (IARC) and WHO. Toxic air pollution poses a greater threat to children, due to their smaller physical size and lung capacity. According to the WHO [4], 4.3 million healthy lives were prematurely lost in 2012 due to indoor air pollution (plus 3.7 million deaths due to outdoor air pollution) and, unfortunately, this number is predicted to increase to 6 million/year by 2050 (this number is doubled if we consider also the deaths caused by outdoor air pollution). Finally, it has been calculated that premature deaths due to air pollution in 2013 cost the global economy about $\$ 225$ billion in lost labor income, or about $\$ 5.11$ trillion in welfare losses worldwide [3].

Therefore, improving air quality has the potential to provide enormous economic, environmental, and health benefits.

\section{Field-Effect Based Gas Sensors for Indoor Air Quality}

Today, there is a number of different chemical sensor technologies which are used for gas sensing applications. They differ in the detection principle, sensitive material, and chemical species detected. Field effect transistors (FETs) are considered highly favorable for gas sensing applications due to their high sensitivity and robustness. Since the introduction of the first field-effect (FE) chemical sensor in 1970 by Bergveld, a lot of progress has been done in terms of material (substrate, insulator, sensing layer) and device development. Silicon carbide $(\mathrm{SiC})$ has been recognized as the leading candidate for FE based sensors due to its compatibility with $\mathrm{Si}$ fabrication technology, and its chemical and physical properties, such as its wide bandgap, stability and chemical inertness.

In the last 15 years, silicon carbide field effect transistors (SiC-FETs) have been widely studied as reliable, cost-efficient, and highperformance gas sensors especially for applications at high temperature in harsh environments such as combustion control or monitoring of car exhaust after-treatment systems [5].

For indoor air quality applications, extremely high sensitivity is required for detection of hazardous key-target indoor air pollutants at the low parts per billion (ppb) concentration level. Moreover, extremely high selectivity is required, due to the presence of other interfering gases in the air at much higher concentrations.

A schematic representation of a SiC-FET sensor and a photo of a mounted sensor chip on a TO8 header together with a Pt100 temperature sensor and a ceramic heater are shown in Fig. 2.

(a)
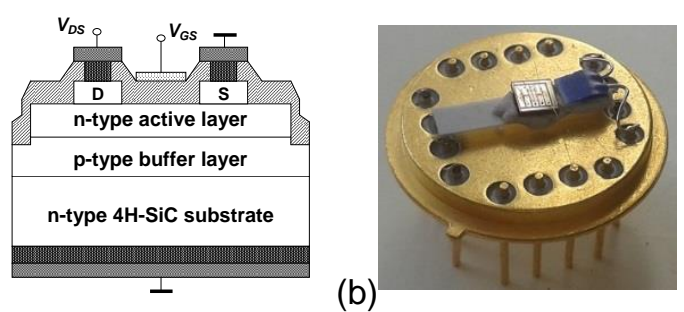

Fig. 2. (a) Schematic of a SiC-FET sensor and (b) mounted sensor chip on a TO8 header.

Within the EU-project SENSIndoor [6], we have developed highly sensitive SiC-FET sensors for detection of three hazardous VOCs, i.e., formaldehyde, benzene, and naphthalene, at the low ppb concentration level down to sub$\mathrm{ppb}$ [7].

We have also demonstrated that a sensing layer composed by a layered structure of a 25 $\mathrm{nm}$ thick, porous, iridium layer, sitting on top of a thin, dense, layer of tungsten trioxide $\left(\mathrm{Ir} / \mathrm{WO}_{3}\right)$ enhances selectivity to naphthalene [8].

Sensor response to 25, 50, and $100 \mathrm{ppb}$ of benzene with an Ir-gated SiC-FET and an Ir $/ \mathrm{WO}_{3}$-gated SiC-FET is shown in Fig. 3. In both measurements, the sensors were operated in the saturation region of the transistor.

In Fig. 3a, the sensor signal is the drain-tosource current, $I_{D S}$, measured by keeping the drain-to-source voltage, $\mathrm{V}_{\mathrm{DS}}$, constant at $4 \mathrm{~V}$, whereas in Fig. $3 b$, the sensor signal is the drain-to-source voltage, $V_{D S}$, measured by keeping the drain-to-source current, $I_{D S}$, constant at $350 \mu \mathrm{A}$.

Operating the device at a constant $V_{D S}$ allows a better control and stability of the sensor signal.

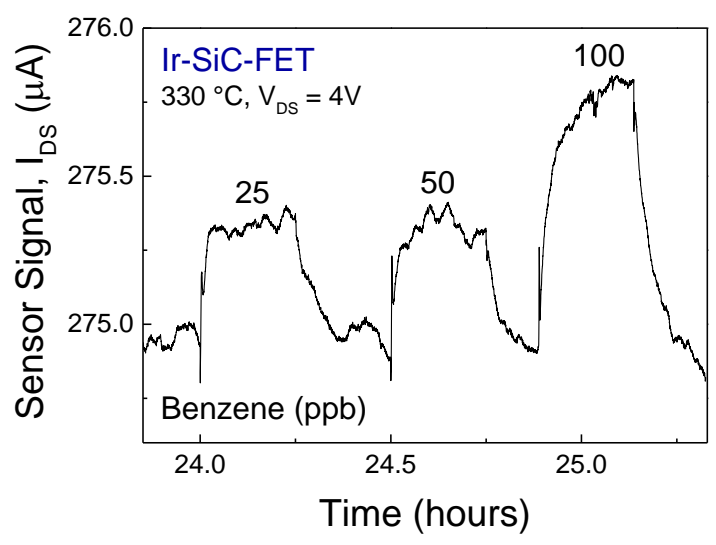

(a) 


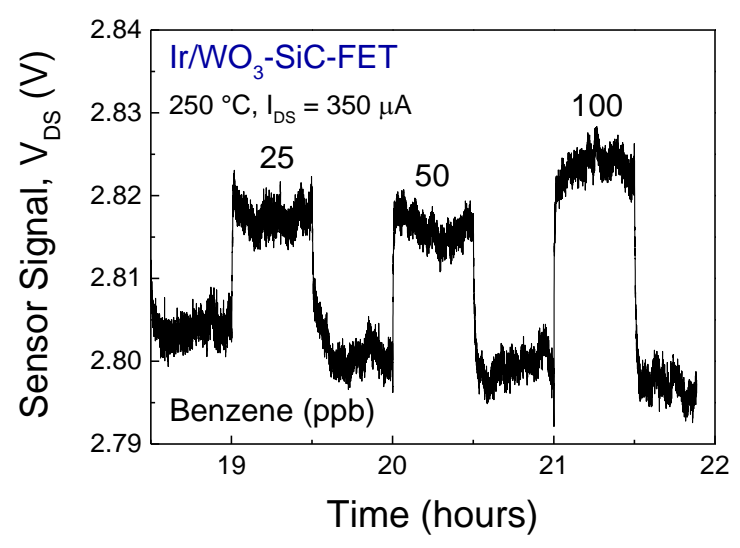

(b)

Fig. 3. Sensor response to 25,50 , and $100 \mathrm{ppb}$ of benzene in saturation region (a) with an Ir-gated SiCFET operated at $330^{\circ} \mathrm{C}$ and (b) with an Ir $/ \mathrm{WO}_{3}$-gated SiC-FET operated at $250{ }^{\circ} \mathrm{C}$.

\section{Conclusion}

Air pollution is a complex issue. In Europe, emissions of many air pollutants have decreased substantially over the past decades, resulting in improved air quality, but concentrations are still too high.

Legislation concerning legal exposure limits is becoming increasingly stringent, whereby effective and user-friendly solutions are urgently needed.

The development of highly sensitive and selective gas sensors based on the SiC-FET technology is a cost- and energy-efficient solution which in the near future will contribute to guarantee a healthy, safe, and comfortable indoor environment through detection of harmful substances at extremely low concentrations below the current legal or recommended exposure levels.

\section{Acknowledgements}

The authors would like to thank the COST Action TD1105 EuNetAir and SenSiC AB, Sweden. This project has received funding from the European Union's Seventh Programme for research, technological development and demonstration, under grant agreement no. 604311 (SENSIndoor).

\section{References}

[1] R. F. Phalen, R. N. Phalen, Introduction to Air Pollution Science. A public health perspective, $1^{\text {st }}$ Edition, Jones \& Bartlett Learning Publ., 2013.

[2] EPA, U.S. Environmental Protection Agency, Technical Overview of Volatile Organic Compounds, last updated on March 17, 2016.

[3] The World Bank and Institute for Health Metrics and Evaluation, 2016. "The Cost of Air Pollution: Strengthening the Economic Case for Action".

[4] WHO, The World Health Organization, 2012.

[5] A. Lloyd Spetz, M. Andersson, R. Pearce, Recent trends in Silicon Carbide ( $\mathrm{SiC}$ ) and Graphene based gas sensors, in: R. Jaaniso and O.K. Tan (Eds.), Semiconductor gas sensors, Woodhead Publishing Series in Electronic and Optical Materials No. 38, 2013.

[6] www.sensindoor.eu.

[7] D. Puglisi, J. Eriksson, C. Bur, A. Schuetze, A. Lloyd Spetz, M. Andersson, Catalytic metal-gate field effect transistors based on SiC for indoor air quality control, Journal of Sensors and Sensor Systems 4, 1-8 (2015); doi:10.5194/jsss-4-1-2015

[8] M. Bastuck, D. Puglisi, J. Huotari, T. Sauerwald, J. Lappalainen, A. Lloyd Spetz, M. Andersson, A. Schütze, Exploring the selectivity of $\mathrm{WO}_{3}$ with iridium catalyst in an ethanol/naphthalene mixture using multivariate statistics, Thin Solid Films (2016) in press; doi: 10/2016/j.tsf.2016.08.002 